San Jose State University

SJSU ScholarWorks

Doctoral Projects

Master's Theses and Graduate Research

Spring 2021

\title{
The Effects of Virtual Reality on Symptom Distress in Patients Undergoing Hematopoietic Stem Cell Transplant
}

\author{
Colleen Vega \\ California State University, Northern California Consortium Doctor of Nursing Practice \\ Robin Whitney \\ Josef Hannah \\ Grant Smith
}

Follow this and additional works at: https://scholarworks.sjsu.edu/etd_doctoral

Part of the Other Nursing Commons

\section{Recommended Citation}

Vega, Colleen; Whitney, Robin; Hannah, Josef; and Smith, Grant, "The Effects of Virtual Reality on Symptom Distress in Patients Undergoing Hematopoietic Stem Cell Transplant" (2021). Doctoral Projects. 137.

DOI: https://doi.org/10.31979/etd.mtyd-8yq7

https://scholarworks.sjsu.edu/etd_doctoral/137

This Doctoral Project is brought to you for free and open access by the Master's Theses and Graduate Research at SJSU ScholarWorks. It has been accepted for inclusion in Doctoral Projects by an authorized administrator of SJSU ScholarWorks. For more information, please contact scholarworks@sjsu.edu. 
Running Head: EFFECTS OF VIRTUAL REALITY ON SYMPTOM MANAGEMENT

The Effects of Virtual Reality on Symptom Distress in Patients Undergoing Hematopoietic Stem Cell Transplant

Colleen Vega, MSN, CNS, ACHPN

Robin Whitney, RN, PhD

Josef Hannah, DO

Grant Smith, MD 
Running Head: EFFECTS OF VIRTUAL REALITY ON SYMPTOM MANAGEMENT

\author{
Abstract \\ The purpose of this QI project was to evaluate the effects of virtual reality (VR) on \\ symptom distress experienced by individuals receiving an allogenic stem cell transplant. \\ Allogenic transplants are associated with a moderate to high risk for distressing symptoms such \\ as depression, anxiety, and pain. VR targets multiple sensory modalities, including auditory, \\ visual or haptic experiences, by using computer-generated scenarios, which can interact with an \\ individual and possibly diminish unpleasant symptoms. Twenty individuals aged 19 to 70 years \\ (median age of 56.5 years) who were hospitalized in an academic setting received VR up to two \\ sessions a week for two weeks. Before and after each session, the participant completed the \\ Edmonton Symptom Assessment Scale Revised (ESAS-r) to evaluate their symptom distress. \\ Paired t-tests were conducted and showed significant improvement in eight out of the ten \\ symptoms addressed in ESAS-r (depression, anxiety, tiredness, drowsiness, appetite, pain, \\ quality of life, and wellbeing). Nausea and shortness of breath had no significant improvements. \\ These findings suggest VR is a novel intervention to treat distressing symptoms in a hospital \\ setting and warrant future investigations exploring VR's impact on prolonged hospitalizations \\ related to distressing symptoms.
}


Running Head: EFFECTS OF VIRTUAL REALITY ON SYMPTOM MANAGEMENT

\section{Introduction}

Hematopoietic stem-cell transplantation (HSCT) in the United States has increased greatly over the last century (Driscoll, Farnia, Kefalas, \& Maziarz, 2017). While the first attempt at stem cell transplant in 1939 was unsuccessful, it has blossomed into an accepted standard of practice for hematologic deficiencies (Léger \& Nevill, 2004). From 2012-2016, there were 42,204 allogenic and 59,229 autologous transplants in the United States (National Marrow Donor Program, n.d.). During the first two to three weeks following HSCT transplant, patients can endure significant stressful stimuli such as mouth sores, pain, nausea and vomiting, diarrhea, appetite changes, fatigue, altered mental status, hair loss, and infection (Frödin, Lotfi, Fomichov, Juliusson, \& Börjeson, 2015; Bevans, Mitchell, \& Marden, 2008). All HSCT patients are at risk for these distressing symptoms during their treatment process and recovery; however, HSCT patients who receive myeloablative conditioning have a higher risk for severe symptoms (Frödin, Lotfi, Fomichov, Juliusson, \& Börjeson, 2015).

\section{Purpose of HSCT}

Hematopoietic stem-cell transplantation (HSCT) aims at prolonging life, improving quality of life, and curing disease in patients with hematologic deficiencies (Driscoll, Farnia, Kefalas, \& Maziarz, 2017). There are three main categories of stem cells transplanted into patients: allogeneic with myeloablative conditioning, allogeneic with nonmyeloablative conditioning, and autologous transplantation (Pei \& Huang, 2019). Individuals receiving an allogenic transplant receive stem cells from a donor who may or may not be related to the patient; while, autologous transplants are stem cells previously collected from the HSCT patient prior to transplant (Marques et al., 2018). HSCT patients who receive myeloablative conditioning are subjected to high doses of chemotherapy to depress their bone marrow function 
Running Head: EFFECTS OF VIRTUAL REALITY ON SYMPTOM MANAGEMENT

prior to a stem cell transplant (Marques et al., 2018). Unfortunately, recipients of stem cells from an allogeneic donor have a 40-60\% risk of developing graft-versus-host disease (Frödin, Lotfi, Fomichov, Juliusson, \& Börjeson, 2015; Villarreal, Alanis, Perez, \& Candiani, 2016). There are numerous physical sequelae from HSCT treatment and graft-versus-host disease: pain, mouth sores, nausea and vomiting, appetite changes, diarrhea, fatigue, hair loss, altered mental status, and infection (Frödin, Lotfi, Fomichov, Juliusson, \& Börjeson, 2015). Complications from an HSCT transplant can range from mucositis to death.

\section{Symptoms}

Psychiatric disturbances can also be prevalent in HSCT patients. Prieto et al. (2002) assessed psychiatric disorders in 1,062 HSCT patients and the impact of these disorders on the hospital length of stay. They found the overall prevalence for any adjustment disorder, anxiety, and mood disorder in the HSCT population was $42.3 \%$. The burden of symptoms identified in Prieto et al.'s study had a negative impact on HSCT patient's comfort and quality of life.

Anxiety and depression can have a negative effect on subjective symptom burden, length of hospital stay, and long-term depression in HSCT patients (El-Jawahri et al., 2015). Kuba et al. (2017) conducted a prospective multicenter study investigating the course of anxiety and depression before and after allogenic stem cell transplant in 239 patients. They found depression rates increased up to 12 to $30 \%$ post HSCT and the long-term effect of depression lasted almost 5 years (Kuba et al., 2017). On the other hand, anxiety rates leveled off at 3 months posttransplantation when compared to the generalized public.

Other prospective studies have also found the risk for depressive symptoms is at the highest-level during hospitalization shortly after transplantation (Kuba et al., 2017; Marques et al., 2018; Seo et al., 2019). Seo et al. (2019) conducted a prospective longitudinal study 
Running Head: EFFECTS OF VIRTUAL REALITY ON SYMPTOM MANAGEMENT

evaluating the psychological symptoms and physical distress experienced by patients following HSCT. They found anxiety and depression scores peaked on the 7th day following transplant with anxiety rates of $27.6 \%$ and depression rate of $36.8 \%$. Aggravating symptoms such as nausea, shortness of breath, lack of appetite, and pain were also associated with increased anxiety (Seo et al., 2019).

An appropriate treatment plan should be created to address the distressing symptoms during the recovery phase of stem cell transplant for HSCT patients to be able to cope with the aftermath of transplant and have a better quality of life. Psychiatric symptoms in HSCT patients are commonly treated with pharmacotherapy such as antidepressants, anxiolytics, and antipsychotics (Amonoo et al., 2019; Bubalo, 2018). However, there are risks which should be considered when starting new medications. These risks include adverse effect profiles, drug-drug interactions, and medical comorbidities (Bubalo, 2018). Commonly used antidepressants, SSRIs and SNRIs, are known to increase the risk of bleeding in HSCT patients due to their effect on platelet function (Amonoo et al., 2019). Increased risk for neutropenia, thrombocytopenia and agranulocytosis are associated with several antipsychotic medications (Amonoo et al., 2019; Nakamura et al., 2019). HSCT patients may also be unable to swallow oral medications if they have severe chemotherapy-induced mucositis, nausea, or vomiting. Most importantly, centrally acting medications, such as opioids, antidepressants, and anxiolytics, can increase the risk profile for delirium in HSCT patients (Nakamura et al., 2019). Delirium in HSCT patients has been shown to lead to poorer neurocognitive functioning, higher levels of fatigue and distress, and greater mortality rate (Nakamura et al., 2019).

Cognitive behavioral therapy (CBT), massage therapy, and music therapy are alternatives to medications for treatment of anxiety and depression. CBT is a behavioral intervention which 
Running Head: EFFECTS OF VIRTUAL REALITY ON SYMPTOM MANAGEMENT

entails psychoeducation on effective coping skills to reduce the stressors associated with HSCT treatment and recovery (Amonoo et al., 2019). While CBT, massage therapy and music are used in clinical practice, there is very limited data investigating the efficacy of these interventions in the HSCT population (Bubalo, 2018). Multiple resources and staff would be required to provide CBT, music, or massage and there could be a financial cost to implement these programs. The prevalence and impact of psychiatric disorders in the HSCT population is well described in literature; however, we still have limited evidence on which interventions provide the most benefit in managing these disorders. Virtual Reality (VR) has the potential to combat the distressing symptoms of pain, depression, and anxiety in HSCT patients while also eliminating the risk factors that are associated with pharmacotherapy.

\section{Virtual Reality}

Virtual reality has been studied in select populations as an intervention to reduce pain, anxiety, and unpleasantness often associated with procedures or treatments (Ioannou, Papastavrou, Avraamides, \& Charalambous, 2020; Ahmad, Bani Mohammad, \& Anshasi, 2020). VR targets multiple sensory modalities, including auditory, visual, or haptic experiences, by using computer-generated scenarios, which can interact with an individual. Headsets or goggles are used to facilitate a perception of reality, which can stimulate the senses of the viewer. The entertaining and immersive effects of VR can be helpful in redirecting a patient's attention from distressing experiences and reduce pain, anxiety, discomfort, or other unpleasantness (Ioannou, Papastavrou, Avraamides, \& Charalambous, 2020; Dascal et al., 2017). With the emerging trend of VR, it is vital VR is studied in specialized populations, such as HSCT, to determine its efficiency. 
Running Head: EFFECTS OF VIRTUAL REALITY ON SYMPTOM MANAGEMENT

Risks and Benefits of VR. Virtual reality can cause some side effects which include perceptuomotor after-effects and simulation sickness, also known as cybersickness (Baniasadi, Ayyoubzadeh, \& Mohammadzadeh, 2020; Kim, Park, Choi, \& Choe, 2018). Individuals with cybersickness can exhibit signs of nausea, vomiting, eye fatigue, dizziness, ataxia, and dizziness. Eye strain and headaches have also been reported in individuals who have prolonged exposure with VR systems (Baniasadi, Ayyoubzadeh, \& Mohammadzadeh, 2020). On the other hand, VR sessions can offer a pleasant distraction and block out bothersome stimuli, including pain or anxiety, which can improve a patient's quality of life (Ahmad, Bani Mohammad, \& Anshasi, 2020).

\section{Theoretical Model}

Kolcaba's Comfort Theory was used as the theoretical foundation for this project. The comfort theory addresses a patient's comfort in the context of the four holistic experiences (environment, psychospiritual, physical, and sociocultural) and can be divided into three parts: health care needs, health-seeking behavior, and institutional integrity (Kolcaba, 2013). First, health care needs, also known as comfort needs, arise when stressful situations occur and can affect a patient's psychospiritual, social, physical, and/or environmental experience. Second, patients who can attain enhanced comfort will improve their health-seeking behavior. Internal behaviors (i.e., cellular level) and external behaviors (i.e., functional capacity, self-care ability, or participation in health programs) are key components of health seeking behaviors. The third part of the comfort theory, institutional integrity, is defined as the quality of care provided by medical teams and can be measured according to length of hospitalization, costs of care, and patient satisfaction (Kolcaba, 2013). The comfort theory assumes all human individuals respond to complex stimuli as a whole person; their responses include physical, psychospiritual, 
Running Head: EFFECTS OF VIRTUAL REALITY ON SYMPTOM MANAGEMENT

sociocultural, and environmental reactions. The whole human response is greater than separate, smaller responses to stimuli. Additionally, all human beings want to be comfortable and will seek comfort whenever possible (Kolcaba, 2013). Kolcaba's comfort theory suggests patients will engage in positive health-seeking behaviors when they are more comfortable.

Comfort theory was applied to the intervention of VR which targeted the distressing symptoms exhibited in the recovery phase of stem cell transplant. VR was used to address the comfort needs of HSCT patients enabling HSCT to achieve their full comfort.

\section{Project Purpose}

The specific aim of this quality improvement project was to determine if virtual reality (VR) can diminish distressing symptoms, such as anxiety and pain scores, in patients undergoing hospitalization for induction chemotherapy and allogenic HSCT. There is emerging data showing cognitive distraction and other relaxing techniques can address symptoms of pain, nausea, and vomiting (Dascal et al., 2017; Kim, 2018; Schneider \& Hood, 2007). VR is one example of a cognitive distraction tool that has effectively been used in a select number of patients to diminish distressing symptoms (Igoannou, Papastavrou, Avraamides, \& Charalambous, 2020; Dascal et al., 2017).

\section{Methods}

\section{Design}

A pretest-posttest quasi-experimental design was used to examine VR as a possible tool to decrease distressing symptoms in HSCT patients undergoing transplantation. Participants who agreed to the QI study were scheduled to receive the VR intervention during their initial hospitalization for transplantation. The within-subjects design was used to control for gender, age, medications (i.e., antiemetics, antidepressants, and opioids), and cancer diagnosis. 
Running Head: EFFECTS OF VIRTUAL REALITY ON SYMPTOM MANAGEMENT

\section{Setting}

The quality improvement project took place in an inpatient setting at an academic health care institution in the Western United States. Subjects were hospitalized in the Blood and Marrow Transplant unit which holds up to forty patients. All subjects were hospitalized for high dose chemotherapy for hematopoietic stem cell transplant and were isolated from other patients in a private room. To prevent the spread of infection, the infection control committee approved virtual reality headsets to be used in the HSCT population if disinfected with hospital approved antiseptic before and after patient use. HSCT staff (i.e.: nurses and physicians) were educated on the quality improvement project through staff meetings and fliers. The project was conducted by the quality improvement project committee that included two advanced practice nurses, one physician, and one researcher.

Sample. Study participants were recruited from an academic health care institution. The study was approved by the human subjects review board of the academic health care institution. Subject enrollment occurred from September 2020 to March 2021. Based on the historical admission rate of patients in a two-month period, we aimed to enroll 20 subjects. A power analysis was not conducted or required since this was a quality improvement project. Inclusion criteria were (a) admittance for induction for HSCT; (b) planned hospitalization for at least 2 weeks; (c) 18 years of age or older; (d) the ability to read and write in English; (e) absence of metastatic or primary disease involving the brain; and (f) no history of seizures, delirium, or motion sickness. VR has the potential to exacerbate neurological dysfunctions such as delirium or motion sickness. Exclusion criteria included any subjects who have tested positive for covid19 infection or waiting to be tested. There was no cost to the subjects to enroll in the QI project. 
Running Head: EFFECTS OF VIRTUAL REALITY ON SYMPTOM MANAGEMENT

\section{Methodologic Approach}

Medical oncologists and HSCT nurses identified any potential participants when they were first admitted to the hospital for transplant. Medical oncologists and HSCT nurses received a 30-minute introduction on the QI project, VR, and how to refer patients to the project. Investigators for the project were trained on data collection and the VR intervention prior to the start of QI project. Every participant was administered the ESAS-r by a trained investigator immediately before and after each VR session. Participants were offered VR up to twice a week for at least 2 weeks. The investigator assisted each participant with placing the headset on to their head, recording the time and date of VR intervention and administering the pre- and posttest of ESAS-r. The project manager collected all data from researchers to file into the JMPS analysis.

Application of VR. A commercially available VR headset (Oculus) was used for application of VR videos. Participants chose from a variety of VR videos from the Samsung and YouTube collection. These videos were categorized as travel, meditation, game, entertainment, sports. During the VR intervention, participants were seated in their hospital bed or chair. Each session lasted up to 20 minutes. Antiemetics, antidepressants, and pain medications prescribed to participants were recorded.

Data Collection. The Edmonton Symptom Assessment Scale Revised (ESAS-r) was used to evaluate symptom distress in HSCT patients. ESAS-r is an analogue scale using 10 items to evaluate symptom distress in patients (Richardson \& Jones, 2009). The ESAS-r is the revised version of ESAS which was created as a clinical tool to assess symptom severity as part of a clinical assessment (Bruera, Kuehn, Miller, Selmser, \& Macmillan, 1991). Changes to the original ESAS included specifying "now" as the time frame for symptoms, improved definitions 
Running Head: EFFECTS OF VIRTUAL REALITY ON SYMPTOM MANAGEMENT

for symptoms, and adding "other symptoms" as a patient specific symptom. Patients rate up to 10 symptoms on the ESAS-r (shortness of breath, nausea, pain, depression, fatigue, drowsiness, anxiety, appetite, quality of life, and well-being $)$ on a $0-10$ scale $(10=$ worst possible symptom, $0=$ no symptom; wellbeing and appetite are rated with $0=$ best symptom). Quality of life was added as the 10th symptom and it has been found to be valid and reliable in the oncology population (Chang, Hwang, \& Feuerman, 2000).

The ESAS-r is a brief survey that requires minimal effort and is easy to complete in a short period of time (Rees, Hardy, Ling, Broadley, \& A'Hern, 1998; Richardson \& Jones, 2009). It is a self-rated scale that has been validated in cancer and palliative care patients (D'Ambruoso et al., 2016; Bruera, Kuehn, Miller, Selmser, \& Macmillan, 1991; Rees, Hardy, Ling, Broadley, \& A'Hern, 1998). The "gold standard" for symptom assessment is the patient's opinion of their symptoms (Rees, Hardy, Ling, Broadley, \& A'Hern, 1998).

Clinical and demographic characteristics of the HSCT participants including ESAS-r scores, cancer diagnosis (ICD-10 codes), sex (male, female, other), race, age (date of birth), prescribed medications (antiemetics, opioids, antidepressants, anxiolytics) and the patient's selected VR video were collected by the project manager after reviewing each participant's medical chart (see table 2). Pre-and-post vital signs (i.e.; respiratory rate and heart rate) and ESAS-r survey scores (shortness of breath, nausea, pain, depression, fatigue, drowsiness, anxiety, appetite, quality of life, and well-being) were documented by the investigator assisting the HSCT participant with VR. Data was managed in an Excel spreadsheet. Each patient was given a unique number and these identifiers were used across datasets.

Analysis. Over a seven-month period, we evaluated the VR intervention. All variables were assessed using descriptive statistics. Means and standard deviations were calculated for 
Running Head: EFFECTS OF VIRTUAL REALITY ON SYMPTOM MANAGEMENT

continuous variables. Categorical variables were displayed as frequencies or percentages. Paired t-tests were used to compare mean ESAS-r scores pre-intervention and post-intervention for each separate encounter. JMPS software was used to format and analyze the data. In all analyses statistical significance was determined by $\mathrm{p}<0.05$.

\section{Findings}

\section{Sample}

The median age of participants was 56.5 years old, and most identified as male (75\%), and non-Hispanic white (70\%). Additional demographic variables are displayed in Table 1 and 2. Majority of the participants watched videos depicting entertainment and travel. Thirty-eight VR interventions were recorded from the sample of twenty participants. Only one participant ended their VR session early due to nausea provoked by watching a VR video. 
Running Head: EFFECTS OF VIRTUAL REALITY ON SYMPTOM MANAGEMENT

Table 1: Demographic results

\begin{tabular}{|lc|}
\hline Variable & N=20 \\
\hline Age (years) & Mean (SD) \\
Sex & $51.5(15.1)$ \\
Female & $\mathrm{N}(\%)$ \\
Male & $5(25 \%)$ \\
& $15(75 \%)$ \\
Race/Ethnicity & $\mathrm{N}(\%)$ \\
Non-Hispanic White & $14(70 \%)$ \\
Latinx/Latino/Latina & $2(10 \%)$ \\
Asian & $2(10 \%)$ \\
Hawaiian & $1(10 \%)$ \\
& \\
Diagnosis & $\mathrm{N}(\%)$ \\
ALL & $5(25 \%)$ \\
AML & $4(20 \%)$ \\
CML & $4(20 \%)$ \\
MDS & $2(10 \%)$ \\
Myelofibrosis & $2(10 \%)$ \\
T-cell Lymphoma & $1(5 \%)$ \\
Multiple Myeloma & $1(5 \%)$ \\
Blastic Neoplasm & $1(5 \%)$ \\
& \\
\hline
\end{tabular}


Running Head: EFFECTS OF VIRTUAL REALITY ON SYMPTOM MANAGEMENT

Table 2: Interventions

\begin{tabular}{lc}
\hline & $\mathrm{N}(\%)$ \\
VR Program & $\mathrm{N}=38$ \\
Travel & $15(39 \%)$ \\
Entertainment & $13(34 \%)$ \\
Relaxation & $7(18 \%)$ \\
Game & $2(5 \%)$ \\
Sports & $1(3 \%)$ \\
& \\
Concurrent & $\mathrm{N}=37$ \\
Medication & \\
Anxiolytic & $12(32 \%)$ \\
Pain Medication & $17(46 \%)$ \\
Antidepressant & $6(16 \%)$ \\
Antiemetic & $27(73 \%)$ \\
\hline
\end{tabular}


Running Head: EFFECTS OF VIRTUAL REALITY ON SYMPTOM MANAGEMENT

\section{Results}

We found significant improvements $(\mathrm{p}<0.05)$ in eight out of ten symptoms addressed by ESAS-r (depression, tiredness, anxiety, drowsiness, lack of appetite, pain, quality of life, and well-being). Lack of appetite (mean difference score of 1.8) and anxiety (mean difference score of 1.15) had the most profound improvement compared to the other six categories $(\mathrm{p}<0.0001)$. Nausea and shortness of breath did not show any statistically significant improvement; however, overall nausea scores appeared to decrease from a score of 1.8 to 1.5 with a mean difference of 0.39. While reduction in nausea scores were not shown to be statistically significant, it did highlight that most participants did not get nauseous when using the VR headset. The total ESAS-r score (scores of the 10 items combined) were also evaluated and we found statistically significant improvements in the total ESAS-r scores $(\mathrm{P}<0.0001$; mean difference score of 10.3). See Table 3 for results. 
Running Head: EFFECTS OF VIRTUAL REALITY ON SYMPTOM MANAGEMENT

Table 3: ESAS-r results

ESAS-r Pre-VR Post-VR Difference P-value

\begin{tabular}{lcccc}
\hline Symptom & $\begin{array}{c}\text { Pre-VR } \\
\text { Score } \\
\text { Mean (SD) }\end{array}$ & $\begin{array}{c}\text { Post-VR Score } \\
\text { Mean (SD) }\end{array}$ & $\begin{array}{c}\text { Difference } \\
\text { Mean (SE) }\end{array}$ & P-Value \\
\hline Pain & $1.4(1.8)$ & $1.0(1.3)$ & $0.4(0.2)$ & 0.03 \\
Shortness of Breath & $0.8(1.5)$ & $0.6(1.1)$ & $0.3(0.1)$ & 0.08 \\
Quality of Life & $3.2(2.6)$ & $2.4(2.4)$ & $1(0.3)$ & 0.0015 \\
Nausea & $1.9(2.0)$ & $1.5(1.7)$ & $0.4(0.3)$ & 0.13 \\
Drowsiness & $2.7(2.5)$ & $1.7(2.1)$ & $1.0(0.3)$ & 0.0003 \\
Depression & $2.5(2.5)$ & $1.5(2.1)$ & $1(0.3)$ & 0.0035 \\
Appetite & $5.0(3.2)$ & $3.2(2.5)$ & $1.8(0.4)$ & $<0.0001$ \\
Anxiety & $2.5(1.8)$ & $1.3(1.8)$ & $1.2(0.2)$ & $<0.0001$ \\
Tiredness & $3.4(2.4)$ & $2.2(2.3)$ & $1.2(0.3)$ & 0.0003 \\
Wellbeing & $3.1(2.5)$ & $2.1(2.1)$ & $1.1(0.4)$ & 0.01 \\
Total ESAS & $26.7(16.0)$ & $17.4(15.0)$ & $10(1.7)$ & $<0.0001$ \\
\hline & & & & \\
\hline & & & &
\end{tabular}


Running Head: EFFECTS OF VIRTUAL REALITY ON SYMPTOM MANAGEMENT

\section{Discussion}

In this quality improvement project examining the impact of VR for patients hospitalized for HSCT, we found the VR intervention was feasible and successful in reducing a variety of distressing symptoms. VR was used in this project to address these symptoms and produced similar results compared to previous VR studies evaluating symptoms in other populations (Chirico, 2016; Ahmad, Bani Mohammad, \& Anshasi, 2020). Statistically significant findings were seen in almost all the ten symptoms evaluated by the ESAS-r and all the symptoms improved by some degree. It is important to emphasize the significant improvement seen in appetite in this project. Lack of appetite has been shown as one of the most common symptoms seen in patients following initial transplant and can remain present for up to six months (Marques et al., 2018). It is unclear why appetite showed significant improvement; however, many of the participants viewed travel and entertainment videos which could have included pictures of food sparking their appetites. Schüssler et al. (2012) found that viewing pictures of food could cause an increase in ghrelin, a hormone that causes hunger.

Overall ESAS-r score also showed substantial improvement in reduction of scores (26.6 vs 16.3) following the VR intervention. Elevated psychological symptoms, such as depression and anxiety, and complications from GVHD (pain, nausea, lack of appetite) have been identified as risk factors for prolonged hospitalization and post-transplantation mortality (Godara, 2021; Solh, 2020). Reducing overall distressing symptoms could translate into reduced hospital costs and length of stay. Only one participant experienced adverse side effects, consistent with previous VR studies showing VR as a well-tolerated intervention. Additionally, a few participants shared that VR was a welcomed escape during the COVID19 pandemic. This project supports the use of VR as an effective, new therapeutic modality for patients undergoing HSCT. 
Running Head: EFFECTS OF VIRTUAL REALITY ON SYMPTOM MANAGEMENT

\section{Limitations}

There are several limitations to this project. This project was conducted during the COVID-19 pandemic which created recruitment difficulties. Some individuals who met criteria for enrollment declined due to concerns the COVID-19 virus could be spread by the VR headset despite having reassurance from infection control that the headset is thoroughly disinfected between participants. Additionally, there were significant reductions in scheduled hospital admissions for HSCT transplants causing the project to be delayed until hospital enrollment increased. Second, the project only investigated transient effects by evaluating symptoms shortly after the VR intervention. It remains to be determined if the observed effect of VR intervention is short-lived or sustainable. Therefore, it would be reasonable to study the sustainability of the effect of VR on periodic interventions throughout the entirety of an individual's hospitalization. Third, the project was designed as a quality improvement project for the transplant unit rather than as a formal research study, making results potentially less generalizable. For instance, we used only a single site with a small number of participants and only examined individuals hospitalized to receive an allogenic HSCT. In addition, our participants identified predominantly as white and male. Future work could extend these results by conducting larger, multisite studies with more diverse participants, employing more rigorous experimental designs (i.e., randomized controlled trials), and examining the effect of VR in patients hospitalized for other conditions. Lastly, the project lacked a standardized timeframe for VR intervention since participants were able to watch anywhere from a few minutes to 20 minutes of VR videos. Participants could also choose to use the VR intervention up to four times in a two-week hospitalization making it difficult to determine whether patients had enough exposure to the 
Running Head: EFFECTS OF VIRTUAL REALITY ON SYMPTOM MANAGEMENT

intervention to produce an effect on symptoms. A longer exposure to VR may be needed to invoke a stronger effect on symptoms.

\section{Nursing Implications}

VR was found to be a distraction intervention that was feasible to implement in the hospital setting. Most of the participants in the project did not report any unusual symptoms, such as headaches, dizziness, or visual disturbances. Despite the minimal side effects, VR should still be used with caution and monitored by staff. Future investigations should continue to explore how VR can impact symptom distress in hospitalized patients and possibly impact hospital costs and length of stay related to distressing symptoms. Additionally, it would be relevant to investigate the effects of repeated exposure to VR to determine if long-term use of VR could have prolonged symptom relief.

\section{Knowledge Translation}

This project found that virtual reality (VR) can diminish distressing symptoms, such as anxiety, depression, and pain scores, in patients undergoing hospitalization for induction chemotherapy and allogenic HSCT. There were minimal side effects seen with VR with only one participant indicating significant nausea when watching a video. A few of the participants indicated videos retrieved from YouTube were blurry which may have negatively influenced the participant's VR experience. Using professional produced videos may improve the VR experience and better impact a patient's distressing symptoms. The findings support the theory that watching VR videos can make hospitalizations more tolerable and possibly improve symptoms; however, clinicians should not always assume symptoms will improve. 
Running Head: EFFECTS OF VIRTUAL REALITY ON SYMPTOM MANAGEMENT

\section{References:}

Ahmad, M., Bani Mohammad, E., \& Anshasi, H. A. (2020). Virtual reality technology for pain and anxiety management among patients with cancer: A systematic review. Pain Management Nursing, 21(6), 601-607. doi:10.1016/j.pmn.2020.04.002

Amonoo, H. L., Massey, C. N., Freedman, M. E., El-Jawahri, A., Vitagliano, H. L., Pirl, W. F., \& Huffman, J. C. (2019). Psychological Considerations in Hematopoietic Stem Cell Transplantation. Psychosomatics, 60(4), 331-342. https://doi.org/10.1016/j.psym.2019.02.004

Baniasadi, T., Ayyoubzadeh, S. M., \& Mohammadzadeh, N. (2020). Challenges and Practical Considerations in Applying Virtual Reality in Medical Education and Treatment. Oman medical journal, 35(3), e125. https://doi.org/10.5001/omj.2020.43

Bruera, E., Kuehn, N., Miller, M.J., Selmser, P., and Macmillan, K. (1991). The Edmonton Symptom Assessment System (ESAS): a simple method for the assessment of palliative care patients. Journal of Palliat Care. 7(2):6-9. DOI:10.1016/j.jpainsymman.2016.10.370

Bubalo J. (2018). Managing the Mental Distress of the Hematopoietic Stem Cell Transplant (HSCT) Patient: a Focus on Delirium. Current hematologic malignancy reports, 13(2), 
Running Head: EFFECTS OF VIRTUAL REALITY ON SYMPTOM MANAGEMENT

109-113. https://doi.org/10.1007/s11899-018-0441-2

Chang, V.T., Hwang, S., and Feuerman, M. (2000). Validation of the Edmonton Symptom

Assessment Scale. Cancer. 88:2164-71. https://doi.org/10.1002/(SICI)1097

0142(20000501)88:9<2164::AID-CNCR24>3.0.CO;2-5

Chirico, A., Lucidi, F., De Laurentiis, M., Milanese, C., Napoli, A. and Giordano, A. (2016),

Virtual Reality in Health System: Beyond Entertainment. A Mini-Review on the Efficacy of VR During Cancer Treatment. J. Cell. Physiol., 231: 275-287. https://doi.org/10.1002/jcp.25117

Cohen, M. Z., Rozmus, C. L., Mendoza, T. R., Padhye, N. S., Neumann, J., Gning, I., ...

Cleeland, C. S. (2012). Symptoms and quality of life in diverse patients undergoing hematopoietic stem cell transplantation. Journal of pain and symptom management, 44(2), 168-180. doi:10.1016/j.jpainsymman.2011.08.011.

Dascal, J., Reid, M., IsHak, W. W., Spiegel, B., Recacho, J., Rosen, B., \& Danovitch, I. (2017).Virtual Reality and Medical Inpatients: A Systematic Review of Randomized, Controlled Trials. Innovations in clinical neuroscience, 14(1-2), 14-21.

D’Ambruoso, S., Walling, A., Hurvitz, S., Drakaki, A., Goldman, J., Pietras, C., Watts, F., 
Running Head: EFFECTS OF VIRTUAL REALITY ON SYMPTOM MANAGEMENT

Coscarelli, A., and Wenger, N. (2016). Use of the Edmonton Symptom Assessment Scale

in patients with advanced cancer referred to an embedded palliative care clinician. Journal of Clinical Oncology.34:26_suppl, 96-96. DOI:

10.1200/jco.2016.34.26_suppl.96

Driscoll, D., Farnia, S., Kefalas, P. and Maziarz, R.T. (2017), Concise Review: The High

Cost of High Tech Medicine: Planning Ahead for Market Access. STEM CELLS

Translational Medicine, 6: 1723-1729. doi:10.1002/sctm.16-0487

El-Jawahri, A., Traeger LN, Kuzmuk K, Eusebio JR, Vandusen HB, Shin JA, et al. (2015).

Quality of life and mood of patients and family caregivers during hospitalization for hematopoietic stem cell transplantation. Cancer. 121:951-959

Frödin, U., Lotfi, K., Fomichov, V., Juliusson, G., \& Börjeson, S. (2015). Frequent and longterm follow-up of health-related quality of life following allogeneic haematopoietic stem cell transplantation. European Journal of Cancer Care, 24(6), 898-910. doi:10.1111/ecc. 12350

Godara, A., Siddiqui, N. S., Munigala, S., Dhawan, R., Kansagra, A. J., Rapoport, A. P., Yared, J. A., \& Dahiya, S. (2021). Length of Stay and Hospital Costs for Patients Undergoing Allogeneic Stem-Cell Transplantation. JCO oncology practice, 17(3), e355e368. https://doi.org/10.1200/OP.20.00170 
Running Head: EFFECTS OF VIRTUAL REALITY ON SYMPTOM MANAGEMENT

Hui, D., and Bruera, D. (2017). The Edmonton Symptom Assessment System 25 years later:

Past, present, and future developments. Journal of Pain and Symptom Management 53(3), 630 - 643. DOI: https://doi.org/10.1016/j.jpainsymman.2016.10.370.

Ioannou, A., Papastavrou, E., Avraamides, M. N., \& Charalambous, A. (2020). Virtual reality and symptoms management of anxiety, depression, fatigue, and pain: A systematic review. SAGE Open Nursing, 6, 237796082093616. doi:10.1177/2377960820936163

Kim, H. K., Park, J., Choi, Y., \& Choe, M. (2018). Virtual reality sickness questionnaire (vrsq): Motion sickness measurement index in a virtual reality environment. Applied Ergonomics, 69, 66-73. doi:10.1016/j.apergo.2017.12.016

Kolcaba, K. (2013). Comfort. In Middle range theories: application to nursing research. (pp. 193-209). Philadelphia: Wolters Kluwer Health.

Kuba, K., Esser, P., Mehnert, A. et al. (2017). Depression and anxiety following hematopoietic stem cell transplantation: a prospective population-based study in Germany. Bone Marrow Transplant 52, 1651-1657. https://doi.org/10.1038/bmt.2017.190

Léger, C. S., \& Nevill, T. J. (2004). Hematopoietic stem cell transplantation: a primer for the primary care physician. CMAJ : Canadian Medical Association journal $=$ journal de l'Association medicale canadienne, 170(10), 1569-1577. doi:10.1503/cmaj.1011625

Marques, A. D., Szczepanik, A. P., Machado, C. A., Santos, P. N., Guimarães, P. R., \& Kalinke, L. P. (2018). Hematopoietic stem cell transplantation and quality of life during the 
Running Head: EFFECTS OF VIRTUAL REALITY ON SYMPTOM MANAGEMENT

first year of treatment. Revista Latino-Americana De Enfermagem, 26(0).

doi:10.1590/1518-8345.2474.3065

Nakamura, Z. M., Nash, R. P., Quillen, L. J., Richardson, D. R., McCall, R. C., \& Park, E. M. (2019). Psychiatric Care in Hematopoietic Stem Cell Transplantation. Psychosomatics, 60(3), 227-237. https://doi.org/10.1016/j.psym.2019.01.005

National Marrow Donor Program, a contractor for the C.W. Bill Young Cell Transplantation

Program operated through the U. S. Department of Health and Human Services, Health Resources and Services Administration, Healthcare Systems Bureau. Donor Registry Transplant Data. Last Updated: June 26, 2019

Pei, X., \& Huang, X. (2019). New approaches in allogeneic transplantation in AML. Seminars in Hematology, 56(2), 147-154. doi: 10.1053/j.seminhematol.2018.08.007

Prieto, J., Blanch, J., Atala, J., Carreras, E., Rovira, M., Cirera, E., \& Castó, C. (2002).

Psychiatric morbidity and impact on hospital length of stay among hematologic cancer

patients receiving stem-cell transplantation. Journal of Clinical

Oncology, 20(7), 1907-1917.

Rees, E., Hardy, J., Ling, J., Broadley, K., \& A'Hern, R. (1998). The use of the Edmonton Symptom Assessment Scale (ESAS) within a palliative care unit in the UK. Palliative Medicine, 12(2), 75-82. 
Running Head: EFFECTS OF VIRTUAL REALITY ON SYMPTOM MANAGEMENT https://doi.org/10.1191/026921698674135173

Richardson, L. A., \& Jones, G. W. (2009). A review of the reliability and validity of the Edmonton Symptom Assessment System. Current oncology (Toronto, Ont.), 16(1), 55. https://doi.org/10.3747/co.v16i1.261

Schneider, S. M., \& Hood, L. E. (2007). Virtual reality: a distraction intervention for chemotherapy. Oncology nursing forum, 34(1), 39-46 https://doi.org/10.1188/07.ONF.39-46

Seo, H. J., Baek, Y. G., Cho, B. S., Kim, T. S., Um, Y. H., \& Chae, J. H. (2019). Anxiety and Depression of the Patients with Hematological Malignancies during Hospitalization for Hematopoietic Stem Cell Transplantation. Psychiatry investigation, 16(10), 751-758. https://doi.org/10.30773/pi.2019.07.12

Solh, M. M., Speckhart, D., Solomon, S. R., Bashey, A., Morris, L. E., Zhang, X., \& Holland, H. K. (2020). The Transplant Evaluation Rating Scale predicts overall survival after allogeneic hematopoietic stem cell transplantation. Blood advances, 4(19), 4812-4821. https://doi-org.laneproxy.stanford.edu/10.1182/bloodadvances.2020002204 
Running Head: EFFECTS OF VIRTUAL REALITY ON SYMPTOM MANAGEMENT 
Running Head: EFFECTS OF VIRTUAL REALITY ON SYMPTOM MANAGEMENT 